\title{
Sächsische Textilwaren im Königreich beider Sizilien Generalhandelsagent Carl Just berichtet aus Neapel nach Dresden (1818-1863)
}

\author{
von \\ DIETMAR STÜBLER
}

\section{Die Einrichtung der sächsischen Generalbandelsagentur in Neapel}

Der Weg, den der Kaufmann Carl Just zurücklegen musste, bevor eine sächsische Handelsvertretung in Neapel, der Hauptstadt des Königreichs beider Sizilien, etabliert werden konnte, und der sächsische König Friedrich August I. ihn zu dessen Leiter ernannte, erwies sich als steinig. Am 9. Juli 1816 regte Just bei Friedrich August I. ein Handelskonsulat in Neapel an, um dadurch zuverlässig auf den sächsischen Handel, besonders auf die Baumwollmanufakturen und auf die Leinenfabriken in der Oberlausitz [...] einen böchst vorteilhaften Einfluß [zu] bewirken. ${ }^{1}$ Der sächsische König fertigte schließlich das Patent für Carl Just als Generalkonsul in Neapel aus und der neapolitanische König erteilte das Exequatur für den in Neapel ansässigen sächsischen Kaufmann. Ferdinand I. musste seine Zustimmung jedoch - auf österreichischen Druck - widerrufen. Immerhin standen Habsburgs Truppen nach dem Sieg über den von Napoleon eingesetzten König Joachim Murat bis August 1817 im festländischen Teil des Königreichs beider Sizilien. Mit Post vom 25. April 1817 erklärte Sachsens Außen- und Innenminister Detlef von Einsiedel Carl Just, in Wien habe der neapolitanische Botschafter dem sächsischen Amtskollegen die Einwände Neapels (de facto Wiens) gegen die Einrichtung eines sächsischen Konsulats in Neapel vorgetragen und stattdessen eine Handelsagentur ins Gespräch gebracht. ${ }^{2}$ In dieser gegenüber dem (General-)Konsulat herabgestuften Form eröffnete Carl Just am 11. Juni 1817 die Generalhandelsagentur Sachsens in Neapel. Eine Instruktion beauftragte ihn, sich um den Schutz des sächsischen Eigentums und der Untertanen des sächsischen Königs zu sorgen und jährlich einen Handelsbericht, nötigenfalls außerordentliche Depeschen, nach Dresden zu schicken. ${ }^{3}$

1 Sächsisches Staatsarchiv - Hauptstaatsarchiv Dresden (im Folgenden: HStA Dresden), 10026 Geheimes Kabinett, 2497/1, Bl. 40; Orthografie und Interpunktion der Archivalien sind dem modernen Standard angenähert. Der Wortbestand blieb unverändert.

2 Vgl. HStA Dresden, 10717 Außenministerium, 3741.

3 Zur Einrichtung der Generalhandelsagentur in Neapel vgl. HStA Dresden, 10736 Ministerium des Innern, 6142. - Archivio Statale di Napoli, Affari Esteri, 3243-3244 Sassonia. 
Die in diesem Artikel ausgewerteten Handelsberichte verwahrt das Hauptstaatsarchiv in Dresden. ${ }^{4}$ Leider gibt es erhebliche Lücken. Es fehlen vor allem die Berichte aus den Jahren 1823 bis 1841, während diejenigen von 1817 bis 1822 und von 1845 bis 1863 fast vollständig archiviert vorliegen. In den Handelsberichten informierte Just über die Marktbedingungen (Absatzchancen für bestimmte sächsische Textilerzeugnisse; Stärke der englischen, französischen und - mit Abstand - der niederländischen Konkurrenten usw.) im festländischen Teil des Königreichs beider Sizilien. Die Insel blieb außerhalb seines Gesichtsfeldes (Schwefelabbau und -vertrieb seit den dreißiger Jahren; Handel mit Wein und Zitrusfrüchten). Die Urteile Justs stützten sich zunächst auf Eindrücke, die er als Augen- und Ohrenzeuge vor Ort gewann. 1848 bis 1860 griff er auf eine aussagekräftige Datenbank zurück: die Statistiken des Hauptzollamtes Neapel. Sie erlaubten es ihm, mengenmäßige (nicht wertmäßige) Angaben über den Import bestimmter Warengruppen aufzulisten. Die Relevanz dieser Quelle erhellt die Tatsache, dass im Hafen von Neapel mehr als $80 \%$ der aus dem Ausland (einschließlich der italienischen Staaten) eingeführten Waren anlandeten. 88,1 \% aller Importzölle des Jahres 1859 kassierte das Königreich beider Sizilien in der Gran Dogana von Neapel; dagegen nur 7,5 \% der Ausfuhrzölle. Hier lagen Lecce (35,4 \%) und Bari (36,1\%) vorn, deren Häfen die Ausfuhren der exportintensiven Regionen Apulien und Kalabrien (Olivenöl, Seide, Wein, Getreide) überwiegend realisierten. ${ }^{5}$

Während Just ziemlich ausführlich über die Möglichkeiten und Notwendigkeiten, über Sicherheit und Wagnis des Textilhandels im Königreich beider Sizilien Bericht erstattete, auch Ernteaussichten und tatsächlich eingefahrenen Erträgen sowie den aktuellen Zolltarifen und Wechselkursen Aufmerksamkeit schenkte, erfuhren die Empfänger seiner jährlichen Handelsberichte nichts über die - sehr bescheidene - Entwicklung des Maschinenbaus, beispielsweise in dem hochgradig subventionierten staatlichen Unternehmen in Pietrarsa (Eisenbahnwaggons, Lokomotiven, Schiffsmaschinen, alles in sehr kleinen Zahlen); über den Schiffbau in Castellamare und Neapel, wodurch sich die Tonnage der Handelsflotte (ohne die sizilianischen Schiffe) zwischen 1818 und 1840 verdoppelte; über die ersten zaghaften Schritte im Eisenbahnbau (Neapel - Caserta; Neapel - Salerno), der

4 Die Entwürfe befinden sich im Bestand des Außenministeriums (10717, Nrn. 37413743); die Ausfertigungen im Bestand des Innenministeriums (10736, Nr. 6142). In diesem Artikel sind ausschließlich die Ausfertigungen der Handelsberichte zitiert. Da sie chronologisch abgelegt sind, erübrigt sich - nach der Nennung des Datums der Ausfertigung im laufenden Text - ein besonderer Ausweis der Fundstellen in den Fußnoten. Zu den außerordentlichen (politischen) Berichten von Carl Just zur Revolution 1820/21 im Königreich beider Sizilien vgl. Dietmar Stübler, Die neapolitanische Revolution (1820/21). Diplomatische Korrespondenzen aus Neapel und Wien, in: Ders., Revolution in Italien. Sächsische Diplomaten und Journalisten über Italien zwischen 1789 und 1871, Leipzig 2010, S. 61-86.

5 Vgl. Augusto Graziani, Il commercio estero del Regno delle Due Sicilie dal 1832 al 1858, in: Archivio economico dell'unificazione italiana, Serie 1, 10 (1960), 1, S. 1-89, hier S. 25 . 
1859 die 100-km-Marke kaum überschritt; oder den vereinzelten Bau von Dampfschiffen, darunter 1817 die Indienstnahme der „Ferdinand II.“, des ersten italienischen Dampfschiffs überhaupt. ${ }^{6}$

Im insularen Teil des neapolitanischen Königreichs richtete Sachsen erst 1855 ein Konsulat ein. ${ }^{7}$ Am 27. Juli 1855 ernannte König Johann den in Messina wohnenden Kaufmann Wilhelm Falkenburg zum Konsul in der Hafenstadt an der Meerenge zwischen Sizilien und dem Festland, wofür ihm Neapels Außenminister Luigi Carafa am 8. Oktober 1855 das Exequatur erteilte. Während des Zusammenbruchs der Bourbonenherrschaft in Neapel hörte Falkenburgs Tätigkeit auf, eine offizielle zu sein und beschränkte sich bis auf weiteres darauf, Berichte zu erstatten und den Untertanen des sächsischen Königs mit Rat beizustehen. ${ }^{8}$ Ihr endgültiges Ende fand die Mission Falkenburgs mit der Ernennung des preußischen Konsuls Giulio Ewald Jäger zum Konsul des Norddeutschen Bundes in Messina. Am 5. Juni 1868 schrieb der Vorsitzende des Gesamtministeriums und Außenminister Paul von Falkenstein an Falkenburg in Messina: Infolgedessen veranlasse ich Sie, Ibre Funktion als Sächsischer Konsul einzustellen. ${ }^{9}$

Die Instruktion vom 27. Juli 1855 verpflichtete auch Falkenburg, jährlich einen Handelsbericht in Dresden einzureichen. Das scheint ihm auch bewusst gewesen zu sein, denn am 7. November 1856 schrieb er an Ferdinand von Beust, er sei im hiesigen Unterzollamt auf Schwierigkeiten gestoßen, zuverlässige statistische Belege zu erhalten, hoffe indessen, solche bald von diesem Jahr zusammenzubringen und die Ebre zu haben, Euer Exzellenz [einen Handelsbericht] zu übersenden. ${ }^{10}$ Die Hoffnung blieb wohl unerfüllt. Jedenfalls findet sich in Dresden kein Handelsbericht aus Messina.

Schließlich postierte der sächsische König Johann in demonstrativer Art und Weise im September 1859 einen Ministerresidenten, Kleist vom Loss, am neapolitanischen Hof. ${ }^{11}$ Als Garibaldis Freischaren vor Neapel standen, floh er zusam-

6 Vgl. Giorgio Candeloro, Storia dell'Italia moderna, Bd. 5: La costruzione dello Stato unitario 1860-1871, Mailand 31970, S. 32-42; Alberto Caracciolo, La storia economica, in: Storia d'Italia, Bd. 3: Dal primo Settecento all'Unità, Turin 1973, S. 511693, hier S. 572-577.

7 Zum sächsischen Konsulat in Messina vgl. die spärliche Überlieferung in: HStA Dresden, 10717 Außenministerium, 3719 und 3720. Eine Erniedrigung zur Handelsagentur wie im Fall von Neapel erschien kaum möglich, nachdem der sächsische König Johann am 7. November 1854 dem Dresdner Bankier Felix Kaskel das Exequatur als Generalkonsul des Königs beider Sizilien in Dresden ausgesprochen hatte. Vgl. Dietmar StüBLER, Giuseppe Garibaldis Freischaren erobern Süditalien. Der sizilische Generalkonsul in Dresden Felix Kaskel engagiert sich für die Bourbonen in Neapel (1854-1860), in: Ders., Revolution in Italien (wie Anm. 4), S. 213-236, hier S. 215-218.

8 HStA Dresden, 10717 Außenministerium, 3719.

9 Ebd.

10 HStA Dresden, 10717 Außenministerium, 3720.

$11 \mathrm{Zu}$ Kleist vom Loss vgl. Dietmar Stübler, Sachsen an der Seite fallender Fürsten. Der sächsische Ministerresident Kleist vom Loss berichtet aus Florenz (1858/59), Neapel/ Gaeta (1859/61) und Rom (1864), in: Ders., Revolution in Italien (wie Anm. 4), S. 237266, hier S. 244-255. 
men mit König Franz II. im September 1860 in die Festung Gaeta und von hier vor dem Bombardement der piemontesischen Artillerie im Februar 1861 nach Rom. Seine politischen Berichte verwahrt das Hauptstaatsarchiv Dresden. Handelsberichte zu schreiben gehörte nicht zu seinen Pflichten.

\section{England beherrscht den neapolitanischen Markt}

Der erste Handelsbericht des sächsischen Generalhandelsagenten Carl Just stammt vom 10. Februar 1818. Er gab einen Überblick des Handels aus dem Königreich Sachsen im Jahre 1817 und den früher verflossenen Jahren mit dem Königreich Beider Sizilien. Darin setzte sich Just mit dem Niedergang der Geschäfte auseinander: In diesen verflossenen Jahren war der Handel aus dem Königreich Sachsen bei weitem geringer als in den früheren und wie sich der englische baumwollne Manufakturbandel immer mehr vergrößerte, so hat sich vorzüglich der Leinwandbandel nach und nach verringert, indem man sich leider an die baumwollnen Stoffe, vorzüglich ibrer Woblfeilheit wegen, so gewöhnt hat, daß selbige die Leinwand beinahe ersetzen. - Vom Jahr 1806 bis 1814 war vorzüglich der Leinwandhandel zwischen beiden Königreichen außerordentlich blübend, allein kaum erschienen englische baumwollne Manufakturwaren, so nabm auch der Leinenhandel ab.

Justs Bericht von Anfang des Jahres 1818 bildete zwei folgenreiche Probleme des sächsischen Außenhandels mit dem Königreich Neapel ab. Nach dem Ende des „napoleonischen Jahrzehnts“ in Italien fielen Kontinentalsperre und Kontinentalsystem fort. Der Zustrom enormer Mengen englischer Textilwaren auf den nunmehr fast ungeschützten süditalienischen Markt überflutete die Nischen, in denen sich die sächsischen Textilfabrikanten niedergelassen hatten. Aus Gründen, die Just auch benannte, waren die englischen Produkte billiger und von höherer Qualität als die der Konkurrenz: Baumwolle kostete in England weniger als andernorts und auch weniger als Schafwolle oder Flachs; außerdem erlaubte der Einsatz von Maschinen mit Dampfantrieb Preise zu bilden, die die Konkurrenz für Schleuderpreise hielt. Und schließlich transportierte die britische Handelsmarine die Waren preiswerter als es die Fuhrwerke der Mitbewerber am Markt auf den beschwerlichen Landwegen vermochten. Die sächsische Handelsroute nach Süditalien zog sich durch Habsburgerlande bis zum österreichischen Freihafen Triest, von dort per Schiff bis Manfredonia an der Adriaküste des neapolitanischen Königreichs und danach noch einmal auf dem Landweg in die Hauptstadt des Südens. ${ }^{12}$

12 Vgl. Günther Meinert, Handelsbeziehungen zwischen Sachsen und Italien 17401814. Eine Quellenveröffentlichung, Weimar 1974, Karte S. 386. Horst Blumberg, Die deutsche Textilindustrie in der industriellen Revolution, Berlin 1965, S. 179 f. 
Auch eine zweite Seite des Umbruchs im Handel zwischen Sachsen und dem Königreich beider Sizilien nach dem Ende der napoleonischen Herrschaft erhellte Just in dem zitierten Handelsbericht vom 10. Februar 1818: die Substitutionskonkurrenz $^{13}$ der Baumwolle gegenüber dem Flachs. Die nachfolgende Tabelle, die sich auf die Gesamtheit der deutschen Länder bezieht, belegt die ursprünglich dominierende Position des Leinengewerbes als wichtigstem Exportzweig der deutschen Gewebefabrikation: ${ }^{14}$

\begin{tabular}{|l|r|r|r|r|r|}
\hline Zweig & 1828 & 1837 & 1850 & 1864 & 1869 \\
\hline & \multicolumn{5}{|c|}{ Textilwarenausfuhr = 100} \\
\hline Leinenwaren & 47 & 28 & 29 & 9 & 10 \\
\hline Seidenwaren & 20 & 15 & 21 & 23 & 32 \\
\hline Baumwollwaren & 9 & 19 & 19 & 13 & 14 \\
\hline Wollwaren & 24 & 38 & 31 & 55 & 44 \\
\hline
\end{tabular}

Die erheblich niedrigeren Löhne, die in Sachsen im Vergleich zu England gezahlt wurden, ${ }^{15}$ verlangsamten den Niedergang der Leinenproduktion gegenüber derjenigen der Woll- und Baumwollindustrie. Verhindern konnten sie ihn nicht. Um dem absehbaren Ruin auszuweichen, spezialisierten sich einige Unternehmen in den sächsischen Hochburgen des Leinengewerbes im Zittauer Raum: Großschönau, Neuschönau, Herrnhut, Sebnitz und in anderen Orten. Mit Blick auf den Export nach Neapel gab es während des gesamten Zeitraums, den wir betrachten, bescheidene, aber immerhin zufriedenstellende Absatzchancen für Matratzenstoffe. Im bereits angeführten Handelsbericht vom 10. Februar 1818 meldete Just: Das Matratzenzeng, [...] welches hauptsächlich in Sebnitz verfertigt wird, behauptet sich, obgleich man es in England in Baumwolle nachmacht, immer noch, und mäßige Partien [...] sind immer gut abzusetzen. Man ziebt es der Baumwolle deswegen vor, weil diese im Sommer sich zu sehr erbitzt, das Leinen bingegen immer kübl bleibt. Ein vergleichbares Beispiel liefern damaszierte Tischtücher. Nach den Angaben in den Handelsberichten aus den fünfziger Jahren verzollte die Gran Dogana in Neapel in der ersten Hälfte des Jahrzehnts pro Jahr im Durchschnitt knapp 450 Stück, in den zweiten fünf Jahren nur noch jährlich knapp 300 Stück aus Sachsen und England. Anders als Just, der jedes Zwischenhoch beim Absatz von Leinenartikeln zum Klimawandel in Richtung Leinenzeug aufsteigerte, betonte die Kaufmannssozietät Zittau in einem Brief vom 23. April 1822 an die

13 Diesen Terminus verwendet Hans-Ulrich Wehler, Deutsche Gesellschaftsgeschichte, Bd. 2: Von der Reformära bis zur industriellen und politischen „Deutschen Doppelrevolution“ 1815-1845/49, München 1987, S. 72.

14 Blumberg, Die deutsche Textilindustrie (wie Anm. 12), S. 289. Die Berechnungen Blumbergs basieren auf den Angaben bei Gerhard Bondi, Deutschlands Außenhandel 1815-1870, Berlin 1958, S. 146. Darauf verweist Blumberg in Anm. 752, S. 289.

15 Vgl. Wehler, Deutsche Gesellschaftsgeschichte (wie Anm. 13), S. 72. 
Landes-Ökonomie-, Manufaktur- und Kommerziendeputation in Dresden, der geringe Warenabsatz in Neapel liegt in der Natur der Dinge, da der Leinenhandel durch die englischen baumwollnen Waren, welche, wie allgemein bekannt, zu beispiellos niedrigen Preisen, man kann mit Recht sagen verschleudert werden, gänzlich und zwar so darnieder gedrückt wird, daß er sich schwerlich in wieder emporheben dürfte. ${ }^{16}$ Das Endgültige des Urteils über das Leinengewerbe, das in diesen Darlegungen mitschwingt, anerkannte Just erst vierzig Jahre später, als er am 26. April 1862 an das Außenministerium in Dresden schrieb: [...] der ehemalige so flotte Leinwandartikel ist von den Engländern ganz verdrängt worden.

Auf einen Artikel, der während des ganzen von uns umspannten Zeitraums anschwellenden Gewinn in die Bilanz der sächsischen Textilfabrikanten einschriftete, lenkte Just von Anfang an die Aufmerksamkeit seiner Adressaten. In dem bereits mehrfach herangezogenen Handelsbericht vom 10. Februar 1818 vermeldete er: Feine Strümpfe von Chemnitz, Glauchau und aus jener Gegend [Meerane, Limbach] haben sich dieses Jahr [1817] sebr gut und corrent verkauft, indem solche weit schöner als die englischen sind, vorzüglich die gestrickten und durchbrochenen für Damen, welche die Engländer, glaube ich, nicht machen, wenigstens kommen keine hierber. Tatsächlich konkurrierten die sächsischen Strumpfwirker seit Beginn der zwanziger Jahre erfolgreich mit den Engländern und verdrängten sie schließlich sogar aus diesem winzigen Segment des neapolitanischen Marktes.

Grosso modo lässt sich sagen: England beherrschte den neapolitanischen Markt. Am Gesamtimport beanspruchte es einen Anteil von $35 \%$; Frankreich $30 \%$; Österreich $8 \%$. In die genannten drei Staaten ging auch der größte Teil des neapolitanischen Exports, nämlich reichlich zwei Drittel. Frankreich lag auf Platz 1 vor Österreich und England. Der Anteil des Handels zwischen den Staaten der Apenninenhalbinsel betrug im erörterten Zeitraum etwa $20 \%$ - mit abnehmender Tendenz. Die Handelsbilanz las sich in all den Jahren stets negativ. Das verursachte in erster Linie der hohe Import von Artikeln der Textil- und Bekleidungsbranche (33\%). Der Export beschränkte sich vorrangig auf Öl (33\% $40 \%$ ), (Roh-)Seide und Getreide, also auf wenige und meistens gering oder gar nicht bearbeitete Rohstoffe. Diese Handelsstruktur erinnert fatal an koloniale Verhältnisse. ${ }^{17}$

16 HStA Dresden, 10736 Innenministerium, 6142, Bl. 47.

17 Vgl. Graziani, Il commercio estero (wie Anm. 5), Tabn. I-VIII; Prospekt D, S. 17; S. 16-27; Nicola Crepas, Le premesse dell'industrializzazione, in: Storia d'Italia, Annali 15: L'industria, hrsg. von Franco Amatori u. a., Turin 1990, S. 127; Angelantonio Spagnoletti, Storia del Regno delle Due Sicilie, Bologna 1997, S. $257-$ 263. 


\section{Rigide Schutzzollpolitik:}

Die neapolitanischen Dekrete aus den Jabren 1823 und 1824

Die Revolution im Königreich beider Sizilien (1./2. Juli 1820 bis 20. März 1821) nahm Carl Just zunächst mit Sympathie auf, die sich steigerte, als er nach Dresden von der Proklamation einer, wie er meinte, monarchischen Verfassung (14. Juli 1820) und der Eröffnung des Parlaments (3. Oktober 1820), dessen Arbeit er voller Interesse verfolgte, berichten konnte: Die Revolution ist zu Ehren und Gloria der Neapolitaner Nation in vier Tagen und zwar obne das geringste Blutvergießen, bis jetzt, abgemacht gewesen [...]. Sobald die Konstitution proklamiert sein wird, werde ich sie Euer Exzellenz gleich mitteilen. ${ }^{18}$

Im Rahmen dieses Artikels interessieren uns die Perspektiven für den Handel mit dem süditalienischen Königreich, die sich Justs Augen eröffneten. Im Handelsbericht vom 6. Januar 1821 schrieb er: Die Revolution hat bestimmt auf den ganzen Handel die allernachteiligsten Folgen gehabt und wird sie so lange haben, als die Drobungen von außen gegen das neue System herrschen; [...] Gründet sich die neue Regierungsform fest und wird sie von den Hoben Mächten anerkannt, wodurch allein die lange und tötende Ungewißheit schwinden kann, so ist vorauszuseben, [...] [dass] man, wie schon die Vorschläge im Parlament gemacht wurden, den Einfubrhandel in Manufakturwaren durch den der Ausfubr der Landesprodukte zu erleichtern suchen [wird], welcher ersterer durch die wirklich unglaublich barten existierenden Gesetze, welche durch die strengen Auslegungen durch die Schikanen der Zollbeamten noch weit unerträglicher sind, und durch die bohen und sebr ungleich verteilten Zölle den rechtlichen Kaufmann nicht nur jede Operation erschwert, sondern auch degoutiert, und die weniger braven zum Kontreband verleitet und somit das Erarium um seine Einkünfte bringt. Es erscheint bemerkenswert, dass Just die Verantwortung für den aktuellen Einbruch des Handels nicht den Revolutionären aufjochte, sondern den in Troppau und später in Laibach versammelten Staaten der Heiligen Allianz, die das Damoklesschwert einer militärischen Intervention über Neapel aufhängten. Könnte sich die Revolutionsregierung hingegen behaupten und die Hohen Mächte von ihren Interventionsplänen abbringen, werde sie ein - aus der Sicht des Freihändlers Just - gerechteres Zollsystem aufrichten und auf diese Weise dem Schmuggel, der erfolgreich mit dem legalen Warenverkehr konkurrierte, die Gewinnaussichten vergällen.

Ende März 1821 fiel das Damoklesschwert: Österreichisches Militär warf die Revolution in Neapel nieder und behielt das Königreich bis 1827 besetzt. Carl Justs Hoffnungen auf eine anwachsende Entwicklung des Handels zerstoben im rauhen Wind der Reaktion, der sich in die Regierungssegel legte.

Im Handelsbericht vom 30. Januar 1822 erschien Carl Just die Revolution nunmehr unselig und infantil. Sie hätte den inländischen und ausländischen Handel

18 Just an Detlef von Einsiedel vom 7. Juli 1821, in: HStA Dresden, 10026 Geheimes Kabinett, 30020/3. Zu den Auffassungen von Carl Just über die neapolitanische Revolution 1820/21 vgl. StüBleR, Die neapolitanische Revolution (wie Anm. 4), S. 61-86. 
total ruiniert. Der Bedarf der Hauptstadt hat sich auf weit mehr denn die Hälfte reduziert, indem das ganze Linienmilitär [wegen seiner Haltung in der Revolution] aufgelöst und die Offiziere auf halben und Drittel Sold gesetzt sind. Der größte Teil der Beamten ist entweder ganz abgedankt oder nur provisorisch beibebalten, und alle jungen Studenten haben in ibre Heimat zurück gemußt. Diese Maßregeln haben also auf den Consumo der Manufakturen im allgemeinen einen unausbleiblich nachteiligen Eindruck, da obige drei Hauptkonsumenten fallen: Die einen haben kein Geld mehr, die andern fallen ganz [...] aus, während das österreichische Besatzungsheer größtenteils von seinen Erbstaaten mit allem versehen [wird], was Manufakturen angebt. ${ }^{19}$

Charakteristisch für den politischen Standort Carl Justs ist die durch den zitierten Handelsbericht vom 30. Januar 1822 verbriefte Tatsache, dass er bereitwillig und zuversichtlich ein Gerücht, das sich bewahrheiten sollte, aufgriff, nämlich: Luigi de'Medici, ein anerkannter guter Financier, werde wieder das Finanzministerium übernehmen. Dieses hatte er bereits 1815 bis zur Revolution 1820 geführt, und als ein vorsichtig konservativer Reformer Respekt bis hinein ins rechtsliberale Lager gewonnen. Aufsehen erregte er, als es ihm gelang, Polizeiminister Antonio Capece Minutolo, Fürst von Canosa, Mitte 1816 aus der Regierung zu drängen. Dessen repressiv-reaktionäre Politik trug aus der Sicht de'Medicis Verantwortung für die überstraffen Spannungen in der neapolitanischen Gesellschaft. Während der Revolution 1820/21 aus der Regierung ausgeschlossen, floh er nach Rom. Nach der Niederwerfung der Revolution schwang sich zunächst der erneut zum Polizeiminister berufene Canosa zum starken Mann auf. Für ihn galten die Häupter der verborgenen Sekten, bald Jakobiner, bald Liberale, bald Carbonari genannt, alle, gleichviel wie sie beißen, als Demagogen und Verbrecher. ${ }^{20}$ Die exzessive Gewaltherrschaft Canosas zwang den neapolitanischen König Ferdinand I., dem Druck Metternichs und Rothschilds nachzugeben, Canosa am 28. Juli 1821 $\mathrm{zu}$ entlassen und 1822 sogar des Landes zu verweisen. Damit war für Luigi de'Medici der Weg frei, wieder das Finanzministerium zu übernehmen, das er bis zu seinem Tod im Jahre 1830 leitete. Nach dem Scheitern seines politischen Konzepts 1820/21 knüpfte er 1822 allerdings nicht dort an, wo die Revolution

19 Der zweite Teil des zitierten Handelsberichts vom 30. Januar 1822 steht im Widerspruch zum bisher Gesagten und ist sichtlich vom Interesse geleitet, dass die Herren Fabrikanten hiesigen Platz nicht ganz aus dem Auge verlieren, weil er ibnen einen bedeutenden Absatz biete. In Entgegnung dazu protestierte die verbitterte Kaufmannssozietät Zittau am 23. April 1822: Justs Bericht sei nicht nur ganz einseitig, sondern auch ganz unrichtig. Der Handlungszustand in Neapel [befinde sich] in trübem Licht. Vgl. HStA Dresden (wie Anm. 16).

20 Aus der Programmatischen Bekanntmachung Canosas an die geliebten Mitbürger vom 15. April 1821, in: Allgemeine politische Annalen, hrsg. von Friedrich Murhard, 3 (1821), 12, S. 451. 
dazwischengetreten war, sondern kehrte die konservative, entschieden antiliberale und antireaktionäre Seite seiner Politik hervor. ${ }^{21}$

Die größte Enttäuschung der hochgestimmten Erwartungen, die Carl Just mit der Revolution und in abgemindertem Maße mit der Rückkehr Luigi de’Medicis in die Regierung verknüpft hatte, bedeuteten die neuen Zolltarife, die die königlichen Dekrete vom 15. Dezember 1823 (provisorisch) und vom 30. November 1824 (definitiv) festlegten. Importierte und exportierte Güter mussten im Rohzustand mit $3 \%$ verzollt werden; im bearbeiteten Zustand dagegen mit $30 \%$. Die erste Kategorie betraf vor allem die Exportartikel des Königreichs beider Sizilien und die aus England eingeführte Baumwolle; die zweite Kategorie die importierten Erzeugnisse der Textilbranche aus England, Frankreich, den Niederlanden und eben auch aus Sachsen. Die Verfügungen der Jahre 1823 und 1824 listeten 239 Titel aus dem Export und 838 Titel aus dem Import des Königreichs Neapel auf. Ein bezeichnender Zusatz begönnerte die neapolitanische Handelsflotte: Für Waren, die auf neapolitanischen Schiffen transportiert wurden, gaben die Zollämter zehnt Prozent Rabatt.22 Just zeigte die neue Situation in Dresden an und auch seine vergebliche Verwendung, um für die betreffenden sächsischen Artikel eine Erleichterung zu erwirken. ${ }^{23}$ Geradezu als Entschuldigung schrieb er, dass auch England und Frankreich, welche am benachteiligsten sind, auf ibre sebr dringenden Vorstellungen durchaus nichts haben erbalten können. ${ }^{24}$

$\mathrm{Zu}$ den Folgen der neuen Zollverordnungen äußerte sich Just rückblickend im Handelsbericht vom September 1843: Die Abnabme der Einfubr ausländischer Manufakturwaren hängt hauptsächlich von den hohen Zöllen [...] ab. Und: Das verleitet zu der unheilvollsten Kontreband, welche bei dem so ausgedehnten Litoral ganz unmöglich zu verhindern ist [...]. - Ganz Puglien wird beinahe mit nichts als Kontreband [...] aus dem Freibafen Triest versehen; die Provinzen Abruzzi von Senigallia und Ancona; Kalabrien von Malta und selbst von Messina; und wir hier [in Neapel] und die naben Provinzen von Marseille, Genua, Livorno und Civitavecchia, lauter Freibäfen. Just nannte das infamen, jede gute Moral tötenden Handel.

Nun stellten hohe Schutzzölle in den zwanziger Jahren kein Alleinstellungsmerkmal des Königreichs beider Sizilien dar. Mit Ausnahme des Großherzogtums Toskana verschanzten sich alle italienischen Staaten hinter ihnen. Die Besonder-

21 Zu Luigi de'Medici vgl. Mauro VAnga in: Dizionario biografico degli Italiani, Bd. 73, Catanzaro 2009, S. 136-140. Zu Antonio Capece Minutolo, principe de Canosa vgl. Alberto Postigliola, in: ebd., Bd. 18, Rom 1975, S. 452-459.

22 Vgl. Graziani, Il commercio estero (wie Anm. 5), S. 9.

23 Einsiedel an Wilhelm von Gutschmid, Direktor der Landes-Ökonomie-, Manufakturund Kommerziendeputation, vom 8. Februar 1824, in: HStA Dresden, 10736 Innenministerium, 6142, Bl. 52. In der Anlage zum Brief befindet sich das im Text genannte Dekret von 1823.

24 Just an Johannes von Minkwitz, Unterstaatssekretär im Außenministerium, vom 13. Januar 1824, in: HStA Dresden, 10026 Geheimes Kabinett, 30020/3, Brief Nr. 12. 
heiten des neapolitanischen Schutzwalls lagen woanders. Erstens war er turmhoch, höher als andernorts. Zweitens sicherte ihn eine abweisende Fußangel, vor der Just im schon zitierten Handelsbericht vom September 1843 die Herren Fabrikanten zu größter Vorsicht mahnte: Das Schlimmste ist, alle Waren, so einmal in biesiger Douane angelangt, können nicht mehr exportiert, müssen die hohen Zölle, nach einem Jahr die Hälfte und das zweite Jabr die andre Hälfte, bezablen und dann hier konsumiert werden. Eine dritte Eigentümlichkeit des neapolitanischen Schutzzolls lag schließlich darin, dass er nicht die in statu nascendi existierende eigene Industrie gegen übermächtige ausländische Konkurrenz abschirmte. Er sollte in den zwanziger Jahren den Aufbau einer nationalen Industrie überhaupt erst einmal in Gang setzen; und seit den dreißiger Jahren zogen ausländische Unternehmer, die sich - staatlich reguliert - in Neapel angesiedelt hatten, Gewinn aus der Handelspolitik des Königreichs beider Sizilien.

Seit Mitte der dreißiger Jahre sahen sich die sächsischen Textilfabrikanten und Kaufleute auf dem neapolitanischen Markt einer zusätzlichen Herausforderung gegenüber: der einheimischen Textilproduktion. Immer öfter tauchten in den Handelsberichten von Carl Just Passagen wie die im September 1843 auf: Wir haben hier drei große baumwollene Spinnereien, welche mit mebreren kleinen gegen 24.000 Zollzentner Garn in ordinären Nummern ${ }^{25}$ jäbrlich liefern. Hingegen kommen aus England [...] ca. 40.000 Zollzentner, so alle Zoll bezablen. Diese Masse von Garn wird auf mechanischen, aber größtenteils Handstüblen, in Waren reduziert und für den inneren Konsum [...] gedruckt, wovon die großen Fabriken von Schlaepfer, Wenner und Co in Salerno gegen 80.000 [...], Meyer und Zollinger in Scafati gegen 25.000 und G. G. Egg in Piedimonte und einige kleinere zusammen circa 12.000 Stück gedruckt auf den Markt bringen. Das übrige wird zu anderen Stoffen von kleinen Fabrikanten verarbeitet [...]. Schon die Namen deuten darauf hin, dass die Firmengründer aus dem Ausland kamen, in der Textilbranche überwiegend aus der Schweiz. Die allerersten zog es noch in napoleonischer Zeit nach Süditalien, zum Beispiel den Züricher Kaufmann Jean-Jacques Egg im Jahre 1812. ${ }^{26}$ Die meisten Schweizer Ansiedlungen stammten aus den dreißiger Jahren des 19. Jahrhunderts: Schlepfer, Wenner und Co 1835; Meyer und Zollinger $1836 .{ }^{27}$

25 Hinsichtlich der Garnqualitäten unterschied man: hochfeine, feine, mittelfeine und gewöhnliche (ordinäre). Sachsen exportierte überwiegend gewöhnliche Garne bzw. Erzeugnisse aus gewöhnlichen Garnen. Vgl. Hubert Kiesewetter, Die Industrialisierung Sachsens. Ein regionalvergleichendes Erklärungsmodell, Stuttgart 2007, S. 364 und Anm. 70, ebd.

$26 \mathrm{Zu}$ den Frühstartern gehörte auch Friedrich Julius Aselmeyer (1809), der mit Carl Just ein Handelshaus in Neapel führte, bis Just per 1. Juli 1819 Zahlungsunfähigkeit anmelden musste.

27 Vgl. Daniela Luigia Caglioti, Vite parallele. Una minoranza protestante nell'Italia del Ottocento, Bologna 2006; Crepas, Le premesse (wie Anm. 17), S. 113. 
Die Schweizer Firmengründer lockten offensichtlich die exorbitant hohen Schutzzölle und das überquellende Reservoir billiger Arbeitskräfte in das Königreich beider Sizilien. Ihre Initiative brachte im Großraum Neapel und im Hinterland von Salerno industrialisierte Zonen hervor, wie sie für Italien in der Mitte des 19. Jahrhunderts typisch gewesen sind. Man denke an Mailand, Genua, Comasco, Valdagno, Prato, Valle del Liri u. a., mit denen sich die küstennahen Zonen um Neapel und Salerno durchaus vergleichen konnten. ${ }^{28}$ Auch im Norden der Apenninenhalbinsel, beispielsweise in der Lombardei und in Piemont, könnten wir zahlreiche starke ausländische Unternehmerdynastien nachweisen. Anders als im Norden feblte jedoch im Süden das Gegengewicht eines zwar bescheidenen, aber immerhin einigermaßen kompakten Unternehmerstandes. ${ }^{29}$ In der Konsequenz integrierten sich die eingewanderten Minderheiten im Norden in die vorgefundene Gesellschaft, während sie sich im Süden bewusst von der neapolitanischen Gesellschaft separierten. Dem diente ganz wesentlich die 1826 in Neapel aus der Taufe gehobene Deutsch-Französische Gemeinde, deren Gründungsmitglied Carl Just gewesen ist. ${ }^{30}$ Über ihre geistig-kulturellen, religiösen und karitativen Aktivitäten berichtete er an keiner Stelle in seinen Handelsberichten. Er beschränkte sich darauf, die sächsischen Fabrikanten darauf aufmerksam zu machen, dass sie neben den hohen Zöllen und der englischen Konkurrenz die erstarkende einheimische Textilproduktion ins Kalkül ziehen müssten, wenn ihre Rechnung aufgehen sollte.

\section{Die Revision der Zolltarife 1845 und 1846 und ibre Folgen für den Handel zwischen dem Königreich Sachsen und dem Königreich beider Sizilien}

Seit Mitte der vierziger, Anfang der fünfziger Jahre brach sich die industrielle Revolution in den deutschen Staaten auf breiter Front und in rasendem Tempo Bahn. Sie verschmolz mit der Revolution des Transportwesens. ${ }^{31}$ In Italien lie $\beta$ die Eisen- und Stablindustrie die große historische Chance vorübergehen, die sich in den industriell fortgeschritteneren und mit Rohstoffen besser ausgestatteten Ländern durch den Eisenbahnbau bot, der den Übergang vom Vorrang der Textilindustrie zu dem der Schwerindustrie und insbesondere zu dem des Metall- und Maschinenbaus bedeutete. ${ }^{32}$

28 Vgl. Luciano Segreto, Storia d'Italia e storia dell'industria, in: Storia d'Italia, Annali 15 (wie Anm. 17), S. 7.

29 Rosario Romeo, Breve storia della grande industria in Italia 1861/1961, Bologna 41974, S. 19.

30 Vgl. Caglioti, Vite parallele (wie Anm. 27), S. 99-128. In Salerno entstand eine entsprechende Gemeinde erst 1862. Vgl. ebd.

31 Vgl. Wehler, Deutsche Gesellschaftsgeschichte (wie Anm. 13), S. 597-614; Dieter ZIEGLer, Die industrielle Revolution, Darmstadt 22009, S. 51-79.

32 Romeo, Breve storia (wie Anm. 29), S. 44 f. 
Diese grundstürzenden Tatsachen spiegelten sich im Handel wider. Die Importe der Staaten des deutschen Zollvereins wuchsen von 1849 bis 1858, im Vergleich zu 1838 bis 1847, um 31 \%, die Exporte sogar um 49 \%. ${ }^{33}$ Anders in Neapel: Die Importe erhöhten sich 1849 bis 1858, gemessen am Jahrzehnt von 1838 bis 1847, um 18 \%, die Exporte im selben Zeitraum nur um 17 \%. ${ }^{34}$ Ins Auge fallen die relativ niedrigen Zuwachsraten der Handelsaktivitäten und das Wachstum des chronischen Defizits der Handelsbilanzen in Neapel. Das Königreich beider Sizilien nahm nur in begrenztem Maße am europäischen Wirtschaftsleben teil. Sein Abstand zu den meisten westeuropäischen Staaten wuchs jetzt rasch. ${ }^{35}$ Ängstlich zögernd versuchten Hof und Regierung durch staatliche Eingriffe die Situation zu bessern. Heraus ragten zwei Zolldekrete aus den Jahren 1845 und 1846, durch die ein Großteil der Importzölle herabgesetzt wurde. Durch den Erlass vom 18. August 1845 fielen die Einfuhrzölle für Nahrungsgüter um $31 \%$ bis $68 \%$. Der Erlass vom 9. März 1846 senkte die Einfuhrzölle für viele Manufaktur- und Industrieerzeugnisse um $15 \%$ bis $60 \%$ ab. ${ }^{36}$ Insgesamt blieben die neapolitanischen Zölle allerdings die höchsten aller italienischen Staaten.

Wie schon 1820 so verband auch 1846 Carl Just die Lockerung der Zollbestimmungen mit hochfliegenden Hoffnungen für die sächsischen Fabrikanten. Ich beeile mich [...] diese wichtige Nachricht für unsern königlich-sächsischen Handel [...] Euer Exzellenz gleich untertänigst anzuzeigen, schrieb Just am 14. März 1846 nach Dresden. Unsere wollnen Waren und vorzüglich feine Tücher können nun wieder besser in Gang kommen, [...]. - Eine günstige Aussicht eröffnet sich unserm Handel in Leinwand aus Bautzen, Zittau, Herrnhut usw., welche wegen der hohen Zölle von 45 Gran der 6/4 breiten, der jetzt auf 24 Gran reduziert ist, gar nicht mehr kommen konnten, von nun an - da der Consumo hauptsächlich für Bettücher war-gewiß aber wieder gesucht werden. Auch die leinenen Drills, so von 45 auf 22 Gran herabgesetzt sind und andere ähnliche Pantalonstoffe gemischt mit Baumwolle werden wieder mehr Abnebmer finden. [...] Auch Strumpfwaren [...] können demnach wieder gegen die biesigen fabrizierten gut besteben.

Während Just sich in seinem Bericht vom 14. März 1846 ein Urteil über den neuen Reduktionstarif für einen folgenden Bericht aufsparte, stand für ihn schon jetzt fest, dass dem alles verderbenden unmoralischen Schleichbandel ein Ziel gesetzt wurde, weil er bei dem größten Teil der vielen Artikel nicht mehr konvenieren kann. Schon ist der Beweis des Zuckers da, denn seit der Zoll auf diesen

33 Die Angaben sind errechnet aus: Bond, Deutschlands Außenhandel (wie Anm. 14), Tab. S. 145.

34 Die Angaben sind errechnet aus: Graziani, Il commercio estero (wie Anm. 5), Tab. 1, S. 48. Carl Justs Handelsberichte aus den fünfziger Jahren bestätigen die Tendenz bei Graziani. Sie belegen sogar, dass die Zolleinnahmen in Neapel während der zweiten Hälfte der fünfziger Jahre niedriger lagen als in der ersten Hälfte dieses Jahrzehnts.

35 Vgl. Giorgio Candeloro, Storia dell'Italia moderna, Bd. 2: Dalla Restaurazione alle Rivoluzione nazionale, Mailand 61973, S. 242 f.

36 Vgl. Graziani, Il commercio estero (wie Anm. 5), S. 9-12. 
reduziert, kömmt in einem Monat mehr im Zollhaus an als sonst in einem Drittel Jahr, [...]. - Was die inländischen Fabriken [der schweizerischen Fabrikanten] anlangt, so bleibt immer noch ein Schutzzoll zwischen 40 à $60 \%$ auf viele Artikel. A la longue sah die Wirklichkeit anders aus, als sie Carl Just, angeschwungen von Begeisterung über das Zolldekret vom 9. März 1846, den sächsischen Fabrikanten in seinem Bericht vom 14. März 1846 vorgespiegelt hatte. In der ersten Hälfte der fünfziger Jahre konnte der sächsische Handel bei ausgewählten Artikeln noch bescheidene Erfolge verbuchen. In seinem Handelsbericht vom 20. Juli 1856 teilte Just mit: Unsere [d. h. die sächsischen] hochfeinen Tuche sind gut gesehen und von den 3058 [in Neapel verzollten] Stücken, angenehmerweise 876 mehr als 1854, kann man ein gutes Dritteil unsern Fabriken zuschreiben. Was übrigens diesen Artikel anbetrifft, sind die hiesigen Manufakturen wenig vorgeschritten, [...]. Unsere Strumpfwaren aus Chemnitz und Limbach haben guten Fortgang und mit vielem Vergnügen habe ich bemerkt, daß unsere Bordenspitzen usw. aus dem Erzgebirge sebr bekannt werden und angenehm sind. - In damaszierten Tafelgedecken ist der Konsum ganz unbedeutend, sowie leider unser ehemaliger florierender Leinwandhandel durch die unglaubliche Woblfeilheit der irischen Leine ganz verdrängt ist.

Zum wiederholten Male erwies sich das gläubige Vertrauen, das Just schon im Handelsbericht vom 14. März 1846 auf die Erholung des sächsischen Leinwandhandels gesetzt hatte, als ungerechtfertigt. Das Flaggschiff der Oberlausitzer Leinenweber, die damaszierten Tafeltücher, erreichte - den gesamten Import in Neapel betrachtet - 1852 und 1853 mit 609 bzw. 556 Stück die besten Werte. Danach wölkten sich die Aussichten ein. Jährlich konnten noch 250 bis 300 Stück abgesetzt werden, überwiegend die billigeren irischen Produkte. Selbst bei den von Just hochgepriesenen Tuchen spürten Fabrikanten und Händler schon in der ersten Hälfte der fünfziger Jahre rückläufige Tendenzen. Vom Gipfel im Jahre 1850 (=3.580 Stück) ging es in der ersten Hälfte der fünfziger Jahre hinunter auf jahresdurchschnittlich 2.150 Stück, um in der zweiten Hälfte der fünfziger Jahre auf jährlich etwa 1.650 Stück abzustürzen. Und außerdem: Der sächsische Anteil an der Gesamtheit der in Neapel verzollten Tuche betrug 1849 und 1850 annähernd zwei Drittel, seit der ersten Hälfte der fünfziger Jahre nur noch etwa ein Drittel am ohnehin geschrumpften Gesamtimport an Tuchen.

Es bleiben die Wirkwaren: Strümpfe, Mützen, Handschuhe usw., und die erzgebirgischen Posamenten: Bänder, Fransen usw. In der ersten Hälfte der fünfziger Jahre passierten die Gran Dogana in Neapel jährlich knapp 50.000 Zollpfund, im zweiten Jahrfünft pro Jahr beinahe die doppelte Menge. Die Handelsberichte von Carl Just wiesen den sächsischen Anteil nur verbal, aber stets rühmend aus. ${ }^{37}$

In dem zitierten Handelsbericht vom 20. Juli 1856 nannte Just zwei Gründe für die zunehmend bescheidenere Stellung der sächsischen Textilfabrikanten und Kaufleute auf dem süditalienischen Markt. Zum einen träten immer mehr Wett-

37 Alle mengenmäßigen Angaben sind aus den Handelsberichten von Carl Just errechnet. 
bewerber auf den Markt. Das galt tatsächlich für die süd- und südwestdeutschen Gebiete (Bayern, Württemberg, Baden), die nach 1840 deutlich gegenüber Sachsen aufholten. ${ }^{38}$ Zum anderen produzierten die fleißigen, großen, durch Schweizer etablierten Firmen im Schutz der immer noch hohen Zollmauern erfolgreicher denn je. Auf mechanischen Dampfstühlen verarbeiteten sie aus England und Nordamerika importierte Gespinste, sodass selbst die englischen, Müblhäuser und Schweizer doch keineswegs wieder ibren ebemaligen großen Verschleiß erringen konnten, den sie einst besaßen und nach den Zolldekreten von 1846 zurückgewinnen wollten. Zwei Naturkatastrophen spitzten die ohnehin bedenkliche Situation, in der sich der neapolitanische Außenhandel befand, erheblich zu. Noch am 10. Juli 1849 schrieb Just nach Dresden: Voriges Jahr war der Weinbau ganz außerordentlich ergiebig, [so] daß man in den Provinzen die Trauben kaum zur Hälfte abnabm und die anderen hängen ließ oder sie preisgab, wer sie nebmen wollte, denn man hatte weder Fässer genug noch lobnte es die Mübe, bei den äußerst geringen Preisen die Weinlese zu vollenden. Doch schon in den ersten fünfziger Jahren zeichnete sich unerwartetes Elend ab. Im Handelsbericht vom 28. Januar 1855 resümierte Just rückblickend: Seit vier Jabren hat die Traubenkrankbeit [Cryptogamie, Blütenlosigkeit der Weinstöcke] so entsetzliche Verbeerung angerichtet, daß die Besitzer der schönsten Weinländer [darunter Carl Just], auch nicht eine einzige Traube zur Reife oder nur vorbringen, vielmebr alles gänzlich verheert und ruiniert betrachten müssen, [...]. Die Provinzen, so am mittelländischen Meere liegen, haben am meisten gelitten, dagegen jene am adriatischen wenig oder nichts, und diese versorgen die Hauptstadt, allein zu einem dreimal höhern Preis als gewöhnlich. Es dauerte annähernd zehn Jahre, bevor der Einsatz von Schwefel die Krankheit bezwang.

Der Heimsuchung der Weinberge auf dem Fuß folgte seit 1852/53 eine Epidemie im Seidenbau. In seinem Handelsbericht vom 20./28. Juli 1856 beschrieb Carl Just das Malheur: Die Umgegend der Hauptstadt, wo die feinste und beste Seide gebaut wird und im Ausland unter dem Namen Royale so geschätzt und beliebt ist, bietet kaum ein Drittel einer mittelmäßigen Ernte dar. Dagegen seien Kalabrien (und die Abruzzen) weit glücklicher gewesen und hatte[n] eine reichliche Seidenernte. Die Ursache der Krankheit (Pebrine, Absterben der Seidenspinnerraupen) bleibe völlig im Dunkeln, weshalb auch niemand wisse, wie ihr abzuhelfen sei. Importe, vorwiegend aus Fernost, traten an die Stelle der fehlenden neapolitanischen Seide.

Eine stabile Komponente des neapolitanischen Außenhandels blieb das Öl, speziell das Olivenöl. Es bildete etwa ein Drittel des Gesamtwertes des Exports. ${ }^{39}$ Abnehmer waren in erster Linie die Staaten nördlich der Alpen, wo die stürmische

38 Vgl. Blumberg, Die deutsche Textilindustrie (wie Anm. 14), Tab. S. 60; Kiesewetter, Die Industrialisierung Sachsens (wie Anm. 25), Stuttgart 2007, Tab. 43, S. 382.

39 Vgl. Crepas, Le premesse (wie Anm. 17), S. 100. 
Entwicklung der Industrie einen großen Bedarf an Öl generierte. Der Versuch, durch eine Revision des Zollsystems und einige andere staatliche Maßnahmen der Wirtschaft Neapels Impulse zu geben, blieben weitestgehend ohne Wirkung. Gegen den Trend schrumpfte der Handelsverkehr zwischen dem Königreich beider Sizilien in den fünfziger Jahren. Neapel fand keinen Anschluß an die von der industriellen Revolution vorwärts getriebene Wirtschaft der Staaten in Westeuropa. Dafür erwies sich die Programmatik zu kurzschrittig und ohne eine angemessene Perspektive. Naturkatastrophen, deren negative Folgen sich nur schwer eingrenzen ließen, taten ein Übriges.

\section{Das Ende des Königreichs beider Sizilien und die Übernabme des sardisch-piemontesischen Zollsystems im Königreich Italien (1860)}

Nach dem Tod König Ferdinands II. (22. Mai 1859) und der Thronbesteigung seines des Regierens unkundigen Sohnes Franz II. ging es mit der Bourbonenherrschaft in Neapel steil bergabwärts. Carl Just kamen einige im Gesamtbereich der Ökonomie liegende Anzeichen der Schlusskrise des Regimes zu Bewusstsein.

1.

Seit der Revolution 1848/49 schwieg er sich über die Staatsfinanzen beharrlich aus. Es sei, so wiederholte er stereotyp, nie etwas amortisiert worden, wie früher immer geschah, noch die Staatsschuld40 oder ein Budget publiziert worden (11. April 1858). Ein Artikel aus dem Dresdner Journal vom 23. März 1858 (Nr. 67, S. 303), der ihm unter die Augen kam, gab ihm Gelegenheit, ein gesellschaftliches Grundübel offenzulegen. In Brüssel, so informierte die Zeitungsnotiz, sei eine Schrift Über die Finanzlage des Königreichs der beiden Sizilien erschienen. Sie beschönige die Lage in Neapel, wenn dort zum Beispiel zu lesen sei: Für den Kredit, welchen der Staat genießt, spricht der hohe Stand der fünfprozentigen Staatspapiere zu 114 und darüber sowie die Blüte der Staatsbank und ibrer Filiale. Der gute Stand der fünfprozentigen Rente sei, so argumentierte Just, in seinem Handelsbericht vom 11. April 1858, kein Beweis des Flors der Finanzen, denn die Ursache davon ist, daß der Neapolitaner Kapitalist nicht weiß, was er mit seinem Geld anfangen soll. Liegende Güter geben wegen der hohen Stenern wenig, zu Unternehmungen oder Spekulationen ist kein Geist vorbanden wie in anderen Ländern [...]. Deswegen kauft der hiesige Bewohner Rente, um sein Geld nicht müßig liegen zu lassen und

40 Im Handelsbericht vom 18. März 1860 schätzte Just die neapolitanische Staatsschuld auf 130 Millionen Dukaten. Tatsächlich betrug sie im März 1861 657,8 Millionen Dukaten, mithin $27 \%$ der Gesamtschuld des eben proklamierten Königreichs Italien. Vgl. Candeloro, Storia (wie Anm. 6), S. 241. 
dies ist die Ursache des hohen Standes der fünfprozentigen Rente. ${ }^{41}$ Hier lenkte Just also die Aufmerksamkeit auf die Umwandlung von Gewinnen aus Handel und Produktion in Staatstitel. Das Kapital blieb passiv. Nördlich der Alpen floss es in Industrieunternehmen, Eisenbahngesellschaften und Handelshäuser. Es wurde aktiv. Anders als in den Industriestaaten Westeuropas formierte sich in Süditalien keine kapitalistische Industriebourgeoisie.

\section{2.}

Einen weiteren Spannungsherd, der zu einer gewaltsamen Entladung drängen konnte, sah Just in der am Ende der fünfziger Jahre einsetzenden Teuerung für Nahrungsgüter. In einem Sonderbericht über den beutigen Stand der Preise der biesigen Landesprodukte schrieb er am 12. August 1858, dass Dampfschiffe Nahrungsgüter wie Fleisch und Geflügel, Obst und Gemüse in ungeheuren Massen nach Marseille transportieren, wo sie zu höheren Preisen verkauft werden können als in Neapel. Als Folge der künstlichen Verknappung der Nahrungsgüter steigen die Preise im Königreich beider Sizilien. Nun interessierte sich für die genannten Lebensmittel nur die mittlere und die privilegierte Oberschicht. Weiterreichende Aufmerksamkeit beanspruchten die Preise für Getreide, darunter besonders für Mais. Im Handelsbericht vom 1./31. Juli 1857 erfasste Just den Ernst der Thematik: Dieses Produkt, so höchst interessant für das Landvolk, da es ibr tägliches Brot und das Hauptfutter der Schweineherden ist, findet sich äußerst hoch im Preise und um solchen zu einer Gleichbeit mit dem der andern Lebensmittel zu bringen, bält die Regierung das Ausfubrverbot fest. Man muß nicht vergessen, daß der Preis des weißen Brotes für die Mittelklasse äußerst wohlfeil ist, unterdessen leidet der Baner bei den enorm hohen Maispreisen Hunger und seine Herden gehen zugrunde. [...] Die konservativen Verfügungen, so die Regierung zum größten Verdruß und Klagen einer Menge unverbesserlicher Börsenspieler und Kornjuden festhält, sind demnach von der größten Moral. Endlich weiß man, daß dieses die persönliche Politik S. M. des Königs ist, [...].

Die staatlich angeordneten und nur selten und für kurze Zeit gelockerten Ausfuhrsperren für Getreide dienten dazu, Vorräte anzulegen, um die Folgen von Missernten einzugrenzen, und die Preistreiberei der großen Produzenten und Händler einzuschränken. Diese hielten die Preise selbst bei gut gefüllten Scheuern hoch, um den Abkauf im eigenen Land zu erschweren und den Verkauf im Ausland, zu Preisen, die im Lande selbst nicht realisiert werden konnten, zu erreichen, wenn sich die heimischen Häfen öffneten. Um das Ausmaß der Spekulation zu veranschaulichen, griff Just im erwähnten Bericht vom 31. Juli 1857 den Preis für einen Tomolo (55,5 Liter) Mais heraus: normal bis zu 90 Gran; aktuell 1,50 Dukaten. Der Regulierungsdruck von Hof und Regierung in Neapel ging gewiss auch von der Furcht vor einer Wiederaufnahme der Revolution von 1848/49 im König-

41 Als die Staatskrise vor dem Umschlag in die Revolution stand, schrieb Just am 18. März 1860 nach Dresden: Die Staatsrenten vier und fünf Prozent sind voriges Jahr bedeutend gewichen und waren manchen Chancen unterworfen. 
reich beider Sizilien aus, eine dunkle Ahnung, die sich sehr schnell im grellen Licht der Wirklichkeit erfüllen sollte. ${ }^{42}$

3.

Drittens schließlich beobachtete Just voller Sorge, wie die raffgierigen Arme der gesamtgesellschaftlichen Krise immer weitere Teile der Gesellschaft umspannten und die soziale Basis der Bourbonenherrschaft verengten. Im Handelsbericht vom 18. März 1860 warnte er die sächsischen Unternehmer: Mebrere und mitunter sebr bedeutende Failliten sind vorgekommen und man befürchtet andere unter diesen Umständen; demnach wiederbole ich den Herren Fabrikanten und Kaufleuten, sich immer nur an die anerkannt soliden Häuser zu wenden, nie Sendungen direkt an Boutiquiers zu machen und nur unter Del Credere mit hiesigem Platz zu arbeiten, welcher immer gefäbrlicher wird.

Aus der Tausendfalt der ungelösten Probleme hatte Just in den vergangenen zwei, drei Jahren wiederholt kleine Ausschnitte belichtet. Nun aber, wenige Wochen vor der Landung der Freischaren Garibaldis auf Sizilien (11. Mai 1860) und ein halbes Jahr vor dem Einmarsch der Rothemden in Neapel (7. September 1860) ging Just über andeutungsvolle Worte hinaus. Er kündigte den Zusammenbruch des zermorschten Bourbonenstaates an: Gewiß ist, daß das verflossene Kriegsjabr hier [in Neapel] sowie überall [auf der Apenninenhalbinsel] öffentliche und private Verluste erzengt hat, allein in anderen Staaten gibt es gute Doktoren und die Wunden beilen und vernarben. Allein bier existiert die einst so berübmte medizinische Fakultät von Salerno nicht mebr, deswegen sind die guten Doktoren verschwunden und die Krankbeit wird immer schlimmer. Aller Handel und Gewerbe liegen ganz darnieder, was auf alle Klassen den empfindlichsten Eindruck macht. Das gemeine Volk schreit und jammert, aber nicht etwa aus aufrübrerischen Absichten, sondern bloß weil der sonstige gute Verdienst mangelt und die ersten Lebensbedürfnisse immer teurer werden und man einer sebr düstern Zukunft entgegengebt.

Unter den militärischen Schlägen der aus Freiwilligen bestehenden Armee des Südens unter dem Kommando Giuseppe Garibaldis und der regulären Armee des Königreichs Sardinien-Piemont fiel die Bourbonenherrschaft in Süditalien zusammen. Am 6. September 1860 flohen Franz II. und sein Hofstaat aus Neapel. Am 17. März 1861 proklamierte das Parlament in Turin das Königreich Italien.

Bereits am 24. September 1860 hatte die sardische Regierung den Zolltarif des Königreichs Sardinien-Piemont im untergegangenen Königreich beider Sizilien eingeführt. ${ }^{43}$ Die Zölle sanken mit einem Schlag um 80 \% und diejenigen zwischen

42 Für die Staaten des Deutschen Bundes am Vorabend der Revolution von 1848/49 erörterte diese Thematik mit vergleichbaren Resultaten: WeHLER, Deutsche Gesellschaftsgeschichte (wie Anm. 13), S. 642-648.

43 Ohne ein Echo waren die Dekrete der Bourbonenregierung vom 1. März, 15. März und 15. Mai 1860, die eine Senkung der Zölle verfügten, verhallt. In der ersten Jahreshälfte 
den Staaten der Apenninenhalbinsel fielen gänzlich weg (Dekret vom 18. Oktober 1860). Den größten Gewinn aus diesen Maßnahmen schlugen dank ihrer marktbeherrschenden Stellung Engländer und Franzosen heraus. ${ }^{44}$ Allerdings ließ er sich in der Gänze nicht sogleich abschöpfen. In seinem Handelsbericht vom 31. Juli 1862 schrieb Just nach Dresden: Im Ganzen hatte sich der Manufakturhandel im verflossenen Jahr nur mit Mühe etwas gebessert. Allein das neuerdings zunehmende Brigantenwesen hat den Provinzbewohnern, so in rubigen Zeiten hier in Neapel ibren Bedarf kauften und jetzt sich nicht getrauen, hierher zu kommen, außerordentlich geschadet und die Geschäfte, wozu auch die Saison beiträgt, sind nicht nur äußerst flau, sondern höchst gedrückt. Der Gran Brigantaggio, die politische Instrumentalisierung des Brigantismus, drohte vorübergehend tatsächlich in eine Konterrevolution umzuschlagen. So etwas war 1799 mit der Armee des Heiligen Glaubens schon einmal gelungen. In der zweiten Jahreshälfte 1861 nahm ein erhebliches Risiko auf sich, wer die gebirgigen Übergänge zwischen Neapel und Bari passieren wollte. Erst der massive Einsatz regulärer Militärverbände des Königreichs Italien warf in der Mitte der sechziger Jahre nach einem opferreichen Kleinkrieg den Gran Brigantaggio nieder.

Über den Tag hinausgesehen erfüllte sich die von Carl Just in der Post vom 31. Juli 1862 gestellte günstige Prognose, dass der gütige Himmel doch auch wieder [rubige Zeiten] geben wird, wo die sächsischen Waren guten Absatz finden. Ein Jahr später, im Handelsbericht vom 30. Mai 1863, klang es schon viel optimistischer: Jedenfalls ist dieses [das neue Zollregime] aber ein wabres Glück für unsere Fabriken. [...] Feine und extrafeine schwarze und Modetuche und geringere Tuche, erstere aus Großenhain usw. usf. und letztere aus Spremberg usw. usf., sind sebr viele hier angekommen und wegen ibrer billigen Preise schnell und gut verkauft worden. Die Leipziger Herren Spekulanten, so von vielen Fabriken ibre Tuche kaufen, sollen bierinnen bedeutend arbeiten. - Was die gebirgischen Artikel anlangt, so ist Glauchau und Meerane ganz besonders in Kleiderstoffen bier angenehm, als: ganz und halb Wolle und auch wohl ganz Baumwolle und wollenen Blumen broschiert. [...] Glatte mit broschierten farbigen Blumen sind außerordentlich gesucht und feblen. Aus Chemnitz Möbelstoffe in ganz Wolle, Halbwolle und Baumwolle sowie halbseidne Kleiderstoffe für Damen sebr begehrt. Strumpfwaren hauptsächlich aus Hohenstein, Grüna und Limbach. Stickerei, Spitzen, Gardinen usw. aus Plawen, Eibenstock, Schneeberg, Annaberg usw. sind angenehm und verkaufen sich gut. - Dieses sind ungefäbr die Waren, welche jetzt zu dem neuen sehr zurückgesetzten Zoll schnellen und guten Absatz hier finden. Auch sind noch andere Orte und Städte wie Zwickau, Oederan, Crimmitschau, Oschatz usw. usf. so in ibren Erzengnissen hier arbeiten.

1860 ging es nicht mehr um Ein- oder Ausfuhrzölle, sondern um Sein oder Nichtsein des Königreichs beider Sizilien.

44 Vgl. Romeo, Breve storia (wie Anm. 29), S. 27 f. 


\section{Die Schließung der sächsischen Konsulate}

Der eben zitierte Handelsbericht vom 29. Januar 1863 ist der letzte einer langen Reihe, die Carl Just nach Dresden gesandt hat. Nach dem für die preußische Armee erfolgreichen Waffengang gegen die österreichischen Heere im „Deutschen Bruderkrieg“ (15. Juni bis 26. Juli 1866) musste Sachsen, das an Österreichs Seite gekämpft hatte, wider Willen dem Norddeutschen Bund beitreten. Am 16. Juni 1868 teilte das Außenministerium in Dresden dem Innenministerium mit, $d a \beta$ die seitherigen diesseitigen Konsuln an genannten Orten [Neapel, Venedig, Genua, Livorno und Messina] angewiesen worden sind, ibre Funktionen einzustellen, sobald die Bundeskonsuln die ibrigen angetreten haben. ${ }^{45}$ In Neapel bezog sich diese Weisung auf den Generalhandelsagenten Carl Just, dessen Aufgaben fortan der preußische Konsul Friedrich Stolte, nunmehr Generalkonsul des Norddeutschen Bundes in Neapel, übernahm.

Das Königreich Sachsen hatte sich seit den frühen dreißiger Jahren daran gewöhnt - wir zitieren die Schreckensvision Metternichs aus dem Jahre 1833 - in wirtschaftlichen Dingen seine Blicke der Furcht wie der Hoffnung nur nach Berlin $z u$ richten, ${ }^{46}$ von wo aus Preußen den Zollverein majorisierte. In der zweiten Hälfte der sechziger Jahre trat ein, was der österreichische Staatskanzler 1833 als letzte Konsequenz vorausgesehen hatte: Sachsen verlor an Preußen auch essenzielle Bestandteile seiner Souveränität: „die volle Verfügung über die Armee; die außenpolitische Dispositionsfreiheit, ja die politische Entscheidungsmöglichkeit bis in recht wesentliche Zweige der öffentlichen Verwaltung. "47 Damit endete das sächsische Konsularwesen, das seit 1815 eine auffallende, nach der Revolution 1848/49 eine sprunghafte Ausweitung erfahren hatte. ${ }^{48}$

45 HStA Dresden, 10736 Innenministerium, 6142, Bl. 163.

46 Metternich an den Kaiser, Wien, Juni 1833, in: Aus Metternich's nachgelassenen Papieren, hrsg. von Richard Metternich-Winneburg, Bd. 5 (= Tl. 2. Friedens-Aera 1816-1848, Bd. 3), Wien 1882, S. 512.

47 Hellmut Kretzschmar, Das sächsische Königtum im 19. Jahrhundert, in: Historische Zeitschrift, 170 (1950), 3, S. 487.

48 Nach den Untersuchungen von Jörg Ludwig gab es um 1800 kein einziges sächsisches Konsulat; 1850 schon 31; 1867 indessen 73 Konsulate. Vgl. JöRg Ludwig, Zur Geschichte des sächsischen Konsulatswesens (1807-1933), in: Consuls et services consulaires au XIX ${ }^{\mathrm{e}}$ siècle, = Die Welt der Konsulate im 19. Jahrhundert, hrsg. von Jörg Ulbert und Lukian Prijac, Hamburg 2010, S. 365-378, hier: Abbildung 1, S. 376. 